\title{
Esmolol Administration for the Treatment of Refractory Ventricular Fibrillation
}

Kazım KARAASLAN ${ }^{1}$, Tarık UMUTOĞLU', Ufuk TOPUZ1, Yasin AY²

${ }^{1}$ Department of Anesthesiology and Reanimation, Bezmialem Vakıf University, İstanbul, Turkey

${ }^{2}$ Department of Cardiovascular Surgery, Bezmialem Vakıf University, İstanbul, Turkey

\section{ABSTRACT}

Ventricular fibrillation (VF) after releasing an aortic cross clamp in patients undergoing open heart surgery procedures is not rare. Ischemia-reperfusion injury after release of the aortic clamp, increased adrenergic tone, and insufficient protection of the myocardium are the possible causes. Amiodarone, lidocaine, and beta blockers have been added to the cardioplegia solutions as a preventive measure for reperfusion VF. We report a case of life-threatening, shock-resistant VF during the weaning period of a cardiopulmonary bypass (CPB) in a 61-year-old male who underwent a mitral valve repair surgery for mitral valve regurgitation. After several defibrillation attempts, refractory VF was turned to normal sinus rhythm shortly after ultra-short acting, beta blocking agent esmolol administration. CPB was terminated successfully following this. In conclusion, VF is still a major problem for clinicians and the treatment of refractory VF is not well defined. In contrast with the absence of the sufficient randomized controlled human studies, theoretically beta blockers could be a choice alternative for shock refractory VF.

Keywords: VF, Esmolol, Refractory, schock resistant, beta blocker

\section{Introduction}

Ventricular fibrillation (VF) after releasing an aortic cross clamp in patients undergoing open heart surgeries is not rare. The possible causes of VF include ischemia-reperfusion injury after the release of the aortic clamp, increased adrenergic tone, and insufficient protection of the myocardium. Immediate defibrillation by direct-current counter shocks is the first choice of treatment. However, ischemia-reperfusion injury complicated with defibrillation damage deteriorates myocardial functions. Amiodarone, lidocaine, and beta-adrenergic blockers have been added to the cardioplegia solutions for the prevention of reperfusion VF (1-4). Beta blockers have many beneficial effects on myocardial cells. They reduce the risk of sudden cardiac death associated with myocardial infarction exceeding $30 \%$. Beta blockers have been reported to reduce VF incidences after acute myocardial infarction (3). Preventive effects of esmolol involve increase in the left ventricular function after beta blocker administration during cardiopulmonary bypass (CPB) or within $10 \mathrm{~min}$ after releasing the aortic clamp (4). Adrenergic blocker usage has shown to decrease the VF incidence in contrast with an increased incidence of a spontaneous return of the heart rhythm without VF (4). Although beta blockers have been reported to have preventive effects against VF, there are limited reports on its effects against refractory VF in humans.

\section{Case Report}

A 61-year-old male patient with mitral regurgitation underwent mitral valve repair surgery. A transthoracic echocardiography imaging before the surgery indicated that he had severe mitral regurgitation and ejection fraction was $48 \%$. The coronary angiography was normal. There were no abnormalities in the whole blood count and biochemical analysis. General anesthesia was induced by etomidate, vecuronium bromide, and fentanyl and patient was intubated. Isoflurane was used to maintain a state of general anesthesia. The patient received fentanyl citrate boluses up to 300-mg boluses 3 times. After median sternotomy, pericardial adhesions were cleared off. The axillary artery and superior and inferior vena cava were cannulated. After aortic cross clamping, heart was arrested 
with antegrade isothermic potassium blood cardioplegia and the cardioplegia solution was intermittently administered. Mitral valve posterior leaflet P2 application and ring annuloplasty repair were performed. The aortic cross clamping time was $34 \mathrm{~min}$. A transesophageal echocardiography (TEE) before weaning from $\mathrm{CPB}$ showed no air bubbles in the left atrium. An arterial blood gas analysis showed no acid-base and electrolyte abnormalities. Patient was normothermic and the hematocrit level was at $29 \%$.

During weaning from $\mathrm{CPB}$, ventricular fibrillation occurred. Immediately, internal defibrillation was performed with a charge of 10 joules. CPB was $50 \%$ of the cardiac output. After every unsuccessful defibrillation attempt, the charge was increased up to 30 joules. After three attempts, amiodarone was intravenously administered in a $300-\mathrm{mg}$ bolus. Following nearly 30 unsuccessful defibrillation attempts, $1 \mathrm{mg} / \mathrm{kg}$ esmolol was intravenously administered. Three min after esmolol administration, the patient was successfully defibrillated with a charge of 30 joules. CBP was terminated successfully after the cessation of refractory VF. TEE performed after weaning from $\mathrm{CPB}$ showed no mitral valve insufficiency. After the surgery, the patient was sent to a postoperative intensive care unit. The patient was extubated $24 \mathrm{~h}$ after the surgery and discharged to the surgical ward after $48 \mathrm{~h}$.

\section{Discussion}

Beta blockers have shown to have many beneficial effects on the heart in both ischemic and non-ischemic conditions. They decrease the oxygen consumption of the myocardium and the incidence of myocardial infarction. They have also shown to decrease VF incidence after myocardial infarction. Further, they have membrane stabilizing and anti-adrenergic effects and increase the VF threshold (3). Animal studies have shown that pre-treatment with beta blockers prior to the induction of VF and cardiopulmonary arrest has an increased rate of return of the spontaneous circulation, increased survival duration and decreased rate of post-resuscitation arrhythmias and myocardial damage. These studies further report that esmolol and standard-dose epinephrine administration during CPR improve resuscitation and increase survival rate in mixed breed pigs and Sprague-Dawley rats with electrically induced $\operatorname{VF}(3,5)$.

There are only few human case reports and case series suggesting that beta blockers have beneficial effects against VF. In an unblinded non-randomized prospective study, storm, 49 patients were divided into two groups. One group received antiarrhythmic drugs in accordance to ACLS Guidelines and the other group was treated with beta blockers. One-week mortality was 4 -fold greater in ACLS group (18 of 22) and one-year overall survival was $5 \%$ and $67 \%$, respectively. This study suggests that sympathetic blockade could be beneficial during the treatment of repeated recurrent VF/VT bouts (6). Also, a retrospective study by Driver et al. (7) concluded that beta blockers should considered in shock-resistant VF patients prior to the termination of CPR efforts. There is no prospective, randomized human trial examining the effects of beta-adrenergic blockers during an ongoing resuscitation of cardiac arrest from VF due to the nature of the condition.

\section{Conclusion}

In conclusion, although VF is still a major problem for clinicians and the treatment of refractory VF is not clear, in absence of sufficient randomized controlled studies, beta blockers could be an alternative for treating shock-refractory VF.

Informed Consent: Written informed consent about all benefical drugs aplication in case of unwanted events was obtained from patient who participated in this study.

Peer-review: Externally peer-reviewed.

Author Contributions: Concept - K.K., T.U.; Design - K.K., U.T.; Supervision - K.K., Y.A.; Funding - K.K., T.U.; Materials - K.K., U.T.; Data Collection and/or Processing - K.K., T.U.; Analysis and/or Interpretation - K.K., U.T.; Literature Review - K.K., T.U.; Writing - K.K., T.U.; Critical Review - K.K., U.T., Y.A.

Conflict of Interest: No conflict of interest was declared by the authors.

Financial Disclosure: The authors declared that this study has received no financial support.

\section{References}

1. Baraka A, Kawkabani N, Dabbous A, Nawfal M, et al. Lidocaine for prevention of reperfusion ventricular fibrillation after release of aortic cross-clamping. J Cardiothorac Vasc Anesth 2000; 14: 5313. [CrossRef]

2. Ayoub CM, Sfeir PM, Bou-Khalil P, Azar M, Haddadin AS, Harfouch D, et al. Prophylactic amiodarone versus lidocaine for prevention of reperfusion ventricular fibrillation after release of aortic cross-clamp. Eur J Anaesthesiol 2009; 26: 1056-60. [CrossRef]

3. Bourque D, Daoust R, Huard V, Charneux M. Beta-Blockers for the treatment of cardiac arrest from ventricular fibrillation? Resuscitation 2007; 75: 434-44. [CrossRef]

4. Sun J, Ding Z, Qian Y. Effect of short-acting beta blocker on the cardiac recovery after cardiopulmonary bypass. J Cardiothorac Surg 2011; 6: 99. [CrossRef] 
5. Killingsworth CR, Wei CC, Dell'Italia LJ, Ardell JL, Kingsley MA, Smith WM, et al. After Prolonged Ventricular Fibrillation ShortActing b-Adrenergic Antagonist Esmolol Given at Reperfusion Improves Survival. Circulation 2004; 109: 2469-74. [CrossRef]

6. Nademanee K, Taylor R, Bailey WE, Rieders DE, Kosar EM. Treating electrical storm: sympathetic blockade versus advanced cardiac life support-guided therapy. Circulation 2000; 102: 742-7. [CrossRef]

7. Driver BE, Debaty G, Plummer DW, Smith SW. Use of esmolol after failure of standard cardiopulmonary resuscitation to treat patients with refractory ventricular fibrillation. Resuscitation 2014; 85: 1337-41. [CrossRef] 